Canadian

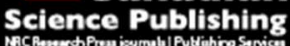

Applied Physiology, Nutrition, and Metabolism Physiologie appliquée, nutrition et métabolisme

\title{
Incidence of Non-Response and Individual Patterns of Response Following Sprint Interval Training
}

\begin{tabular}{|r|l|}
\hline Journal: & Applied Physiology, Nutrition, and Metabolism \\
\hline Manuscript ID & apnm-2015-0449.R1 \\
\hline Manuscript Type: & Article \\
\hline Date Submitted by the Author: & 08-Oct-2015 \\
\hline Complete List of Authors: & $\begin{array}{l}\text { Gurd, Brendon J.; Queens University, } \\
\text { Giles, Matthew; Queens University } \\
\text { Bonafiglia, Jacob; Queen's University, School of Kinesiology and Health } \\
\text { Studies } \\
\text { Raleigh, James; Queen's University, Kinesiology } \\
\text { Boyd, John; Queen's University, School of Kinesiology and Health Studies } \\
\text { Ma, Jasmin; Queen's University, School of Kinesiology and Health Studies } \\
\text { Zelt, Jason; Queen's University, School of Kinesiology and Health Studies } \\
\text { Scribbans, Trisha; Queen's University, School of Kinesiology and Health } \\
\text { Studies }\end{array}$ \\
\hline Keyword: & $\begin{array}{l}\text { aerobic evaluation, exercise training < exercise, exercise intensity }< \\
\text { exercise, exercise physiology < exercise, exercise performance < exercise }\end{array}$ \\
\hline
\end{tabular}


Incidence of Non-Response and Individual Patterns of Response Following Sprint Interval Training

Brendon J. Gurd, Matthew D. Giles, Jacob T. Bonafiglia, James P. Raleigh, John C. Boyd, Jasmin K. Ma, Jason G.E. Zelt and Trisha D. Scribbans

School of Kinesiology and Health Studies, Queen's University, Kingston, ON K7L 3N6, Canada

M. Giles:9mgd2@queensu.ca,

J. Bonafiglia: 0jtb1@queensu.ca,

J. Raleigh: 9jr34@queensu.ca,

J. Boyd: c.boyd@dal.ca,

J.Ma:8jkm@queensu.ca,

J.Zelt: jasonzelt@gmail.com,

T.Scribbans:0ts5@queensu.ca.

Corresponding Author:

Brendon J. Gurd, PhD

Telephone: 613-533-6000 ext.79023

Fax: 613-533-6000

Email: gurdb@queensu.ca 


\begin{abstract}
The current study sought to explore the incidence of non-responders for maximal or submaximal performance following a variety of sprint interval training (SIT) protocols. Data from 63 young adults from 5 previously published studies were utilized in the current analysis. Non-responders were identified using 2 times the typical error (TE) of measurement for $\mathrm{VO}_{2}$ peak $(2 \times \mathrm{TE}=1.74 \mathrm{~mL} / \mathrm{kg} / \mathrm{min})$, lactate threshold $(2 \times \mathrm{TE}=15.7 \mathrm{~W})$, or $500 \mathrm{kcal}$ time to completion $(\mathrm{TTC} ; 2 \times \mathrm{TE}=306$ secs $)$ trial. TE was determined on separate groups of participants by calculating the test re-test variance for each outcome. The overall rate of nonresponders for $\mathrm{VO}_{2}$ peak across all participants studied was 22\% (14/63) with 4 adverse responders observed. No non-responders for $\mathrm{VO}_{2}$ peak were observed in studies where participants trained 4 times per week $(n=18)$, while higher rates were observed in most studies requiring training 3 times per week $(30-50 \% ; n=45)$. A non-response rate of $44 \%(8 / 18)$ and $50 \%$ $(11 / 22)$ was observed for the TTC test and lactate threshold, respectively. No significant correlations were observed between the changes in $\mathrm{VO}_{2}$ peak and TTC $(\mathrm{r}=0.014 ; \mathrm{p}=0.96)$ or lactate threshold $(\mathrm{r}=0.17 ; \mathrm{p}=0.44)$. The current analysis demonstrates a significant incidence of non-responders for $\mathrm{VO}_{2}$ peak and heterogeneity in the individual patterns of response following SIT. Additionally, these data support the importance of training dose and suggest that the incidence of non-response may mitigated by utilizing the optimal dose of SIT.
\end{abstract}

Key words: High-intensity interval training (HIIT), exercise dose, $\mathrm{VO}_{2}$ peak, lactate threshold, submaximal performance, time trial, non-responder, adverse responder, typical error, individual response. 


\section{Introduction}

Sprint interval training (SIT), a subclass of high intensity interval training (HIIT) requiring participants to complete brief maximal efforts separated by periods of rest (Sloth et al. 2013), has garnered recent attention as a time efficient alternative to endurance training (END) (Gillen and Gibala 2014). On a group level, SIT induces similar improvements to END for $\mathrm{VO}_{2}$ peak (Gist et al. 2013, Sloth et al. 2013), lactate threshold (Esfarjani and Laursen 2007, Zelt et al. 2014), and submaximal performance (Gibala et al. 2006, MacPherson et al. 2011, Scribbans et al. 2014a). While our group, and others, have repeatedly demonstrated the benefits of both SIT and END at a group level, there is increasing evidence that there is substantial heterogeneity in how individual participants respond to exercise training.

Bouchard et al. (1999) demonstrated a heterogeneity in changes in aerobic capacity ( $\mathrm{VO}_{2}$ peak) following END with some participants exhibiting large improvements and others experienced minimal or no gains. Non-responses to END has also been observed for indices of submaximal performance (anaerobic/lactate threshold, submaximal exercise heart rate, time trial performance) (Vollaard et al. 2009, Scharhag-Rosenberger et al. 2012). Although recent estimates suggest the incidence of non-responders to END for $\mathrm{VO}_{2}$ peak is between 20 and $45 \%$ (Sisson et al. 2009) it is important to note that there are few individuals who do not respond positively to END by either increasing maximal ( $\mathrm{VO}_{2}$ peak), or submaximal performance (Vollaard et al. 2009, Scharhag-Rosenberger et al. 2012).

A recent meta-analysis suggests that the individual $\mathrm{VO}_{2}$ peak responses to HIIT are likely comparable, if not superior to those observed following END (Bacon et al. 2013). Accordingly, it has been hypothesized that higher intensities of exercise may induce adaptation in individuals with low sensitivity to END (Buford et al. 2013). While these studies suggest that the high 
exercise intensities associated with SIT may induce adaptations in more individuals, nonresponders for $\mathrm{VO}_{2}$ peak following SIT have been observed following brief ( 6 training sessions over 2 weeks) SIT protocols (Astorino and Schubert 2014). Individual patterns of response across $\mathrm{VO}_{2}$ peak and indices of submaximal performance were observed (Astorino and Schubert 2014). Importantly, this study identified non-responders using previously published co-efficient of variations, an approach that has also been utilized by others (Scharhag-Rosenberger et al. 2010), but fails to consider both biological and technical error within the lab examining the rates of response to training. Recently, more critical approaches to determining cut-points for identifying non-responders that consider both biological and technical error, and should minimize the risk of misidentifying responders as non-responders and vis versa, have been proposed (Hopkins 2000, Bouchard et al. 2012). Thus, despite the substantial evidence supporting the efficacy of SIT on a group level, the incidence of non-responders for maximal $\left(\mathrm{VO}_{2}\right.$ peak) and submaximal performance has not been established using statistically sound cutpoints, nor has the incidence of non-response and individual patterns of response been examined following training interventions lasting more than 2 weeks.

As the awareness of the individual variability in the responsiveness to END grows, and SIT continues to increase in popularity, there is a need for more information regarding the rates of non-response following SIT such that we can move towards personal/optimal exercise prescription. Thus, the primary purpose of the current study was to examine the incidence of non-response in $\mathrm{VO}_{2}$ peak across a range of SIT protocols ranging from 3-6 weeks in duration utilizing a critically established cut-point for the identification of non-responders. Individual patterns of non-response were also examined following SIT by comparing individual changes in $\mathrm{VO}_{2}$ peak with changes in lactate threshold or submaximal performance. 


\section{Materials and Methods}

Data from a total of 63 young adults from 5 previously published studies (Boyd et al. 2013, Ma et al. 2013, Scribbans et al. 2014a, 2014b, Zelt et al. 2014) were utilized in the current analysis (all 5 studies are described briefly below). Novel experiments were also conducted in order to determine the typical error (TE) of measurement for $\mathrm{VO}_{2}$ peak, lactate threshold, and a $500 \mathrm{kcal}$ time to completion (TTC) trial. All experimental procedures performed were approved by the Health Science Human Research Ethics Board at Queen's University and confirmed to the Declaration of Helsinki. Verbal and written explanation of the experimental protocols and associated risks were provided to all participants prior to their provision of written informed consent.

\section{Data Collection for Previously Published Studies}

For all studies, $\mathrm{VO}_{2}$ peak was determined using an incremental ramp test to volitional fatigue performed on a cycle ergometer (Monark, Ergomedic 874E, Varberg, Sweden) with gas exchange being collected throughout the test using a metabolic cart (Moxus, AEI technologies, Pittsburgh, PA). This protocol has been described in detail elsewhere (Edgett et al. 2013a). Two studies utilized a 500 kcal time to completion (TTC) trial (Boyd et al. 2013, Scribbans et al. 2014a) that required participants to cycle at a self-selected cadence against a load expected to elicit 50\% of $\mathrm{VO}_{2}$ peak work rate at 60 PRM (Jeukendrup et al. 1996). One study (Zelt et al. 2014) calculated lactate threshold using the first recorded work rate with lactate $>4 \mathrm{mmol} / \mathrm{L}$ method (Bishop et al. 1998) from fingertip capillary blood samples collected within the last 10 seconds of each step of the $\mathrm{VO}_{2}$ peak protocol. Lactate was analyzed using a Lactate Scout + (EFK Diagnostics, Magdeberg, Germany). 
Boyd et al., 2013

One subset of the participants from this study was utilized in the current analysis. This subset consisted of overweight/obese, sedentary men ( $\mathrm{n}=9$; age, 22.7 $\pm 3.8 \mathrm{yrs}$; BMI, $32.3 \pm 1.9$ $\mathrm{kg} / \mathrm{m}^{2} ; \mathrm{VO}_{2}$ peak, $\left.35.4 \pm 5.7 \mathrm{~mL} / \mathrm{kg} / \mathrm{min}\right) . \mathrm{VO}_{2}$ peak and TTC trials were performed before and after training. Training consisted of 3 sessions per week for 3 weeks, utilizing an interval protocol of 8-10 1-minute intervals at $\sim 100 \%$ maximal aerobic power separated by 1 minute of loadless cycling.

Ma et al., 2013

Recreationally active men ( $\mathrm{n}=8$; age, $20.6 \pm 1.6 \mathrm{yrs}$; BMI, $24.6 \pm 1.0 \mathrm{~kg} / \mathrm{m}^{2} ; \mathrm{VO}_{2}$ peak, $40.5 \pm 3.8 \mathrm{~mL} / \mathrm{kg} / \mathrm{min}$ ) trained 4 times per week with $\mathrm{VO}_{2}$ peak being measured before and after 4 weeks of training. Training consisted of 8, 20 second intervals targeting $170 \%$ of peak aerobic power separated by 10 seconds of rest for a total training duration of 4 minutes.

Scribbans et al., 2014a

One subset of recreationally active participants from this study were used in the current analysis. This subset ( $\mathrm{n}=10 ; 2$ female; age, 21.0 $\pm 2.0 \mathrm{yrs}$; BMI, 23.0 \pm 3.9 ; $\mathrm{VO}_{2}$ peak, $48.3 \pm 6.1$ $\mathrm{mL} / \mathrm{kg} / \mathrm{min}$ ) trained 4 times per week with $\mathrm{VO}_{2}$ peak and TTC trials being performed before and after 6 weeks of training. Training consisted of 8,20 -second intervals targeting $\sim 170 \%$ of peak aerobic power separated by 10 seconds of rest for a total training duration of 4 minutes. Scribbans et al., 2014b

This study compared the impact of Resveratrol supplementation on training induced increases in $\mathrm{VO}_{2}$ peak. Because no training induced differences in $\mathrm{VO}_{2}$ peak were observed between conditions, data from all participants (placebo and Resveratrol) completing both preand post-training $\mathrm{VO}_{2}$ peak tests, and the corresponding time to completion test has been included 
in the current analysis. Recreationally active men $(n=14$; age, 21.6 \pm 1.0 yrs; BMI, 23.3 \pm 2.2 ; $\mathrm{VO}_{2}$ peak, $50.0 \pm 5.6 \mathrm{~mL} / \mathrm{kg} / \mathrm{min}$ ) trained 3 times per week for 4 weeks and $\mathrm{VO}_{2}$ peak was measured before and after training. Training consisted of 8,20 -second intervals targeting $\sim 170 \%$ of peak aerobic power separated by 10 seconds of rest for a total training duration of 4 minutes.

Zelt et al., 2014

Two subsets of recreationally active male participants from this study were used in the current analysis. Both subsets trained 3 times per week for 4 weeks with $\mathrm{VO}_{2}$ peak and lactate threshold measured before and after training. The first subset $(n=12 ;$ age, 22.0 \pm 2.0 yrs; BMI, $26.0 \pm 3.0 \mathrm{~kg} / \mathrm{m}^{2} ; \mathrm{VO}_{2}$ peak, $44.2 \pm 8.7 \mathrm{~mL} / \mathrm{kg} / \mathrm{min}$ ) performed 4-6, 15 -second intervals per training session. The second subset ( $\mathrm{n}=10$; age, $23.0 \pm 5.0 \mathrm{yrs}$; BMI, $25.0 \pm 3.0 \mathrm{~kg} / \mathrm{m}^{2}$; $\mathrm{VO}_{2}$ peak, $49.8 \pm 7.8$ $\mathrm{mL} / \mathrm{min}$ ) performed 4-6, 30-second intervals per training sessions. All intervals for both subsets required an all-out effort and were performed against a resistance equal to $7.5 \%$ body weight. Determination of Typical Error

In order to determine $\mathrm{TE}$ for $\mathrm{VO}_{2}$ peak and lactate threshold, 8 recreationally active participants (age, $21.0 \pm 0.8 \mathrm{yrs}$; BMI, $21.0 \pm 2.3 \mathrm{~kg} / \mathrm{m}^{2}$; $\mathrm{VO}_{2}$ peak, $44.4 \pm 5.6 \mathrm{~mL} / \mathrm{kg} / \mathrm{min}$ ) reported to the lab on 2 separate occasions separated by at least a week. On each visit to the lab participants performed identical incremental ramp tests to volitional fatigue as described above and reported in detail previously (Edgett et al. 2013b, Zelt et al. 2014). Both $\mathrm{VO}_{2}$ peak and lactate threshold were determined for each test and the resulting values were utilized to calculate TE. A similar experiment was performed to determine TE for the $500 \mathrm{kcal}$ time to completion (TTC) trial. Fifteen recreationally active individuals (age, 19.2 \pm 0.9 yrs; BMI, 24.0 \pm 2.8 ; $\mathrm{VO}_{2}$ peak, $42.9 \pm 8.2 \mathrm{~mL} / \mathrm{kg} / \mathrm{min}$ ) reported to the lab on 3 separate occasions. During their first 
visit participants performed a $\mathrm{VO}_{2}$ peak test to determine the appropriate work rate for the subsequent TTC tests. On the 2 subsequent visits, which were separated by at least a week, participants performed a $500 \mathrm{kcal}$ TTC test as described above and reported previously (Boyd et al. 2013, Scribbans et al. 2014a). The TTC for each test was used to calculate TE for the TTC test.

Typical error (TE) of measurement was calculated for $\mathrm{VO}_{2}$ peak, lactate threshold and TTC as described previously (Hopkins 2000$)$ utilizing the following equation $\left(\mathrm{TE}=\mathrm{SD}_{\mathrm{diff}} / \sqrt{ } 2\right)$ :

$$
T E=S D_{\text {diff }} / \sqrt{2}
$$

Where $S D_{\text {diff }}$ is the variance (standard deviation) of the difference scores observed between the 2 repeats of each test. A non-responder for $\mathrm{VO}_{2}$ peak, lactate threshold or TTC was defined as an individual who failed to demonstrate an increase or decrease that was greater than $2 \mathrm{x}$ the TE away from 0 . The odds of an individual change that is greater than $2 \mathrm{x}$ the TE being a true physiological change are 12:1 (Hopkins 2000).

\section{Statistical Analysis}

Data are expressed as means and standard deviations. Changes in $\mathrm{VO}_{2}$ peak, lactate threshold and TTC tests at the group level within individual studies were analyzed using paired t-tests. Relationships between the response in $\mathrm{VO}_{2}$ peak and either lactate threshold or TTC was assessed using the Pearson correlation coefficient as was the relationship between baseline VO2peak and the change in $\mathrm{VO}_{2}$ peak following training.

\section{Results}

Typical error $(\mathrm{TE})$ was $\pm 0.87(2 \times \mathrm{TE}=1.74) \mathrm{mL} / \mathrm{kg} / \mathrm{min}$ for $\mathrm{VO}_{2}$ peak, $\pm 7.8(2 \times \mathrm{TE}=15.7)$ watts for lactate threshold and $\pm 153(2 \mathrm{x} \mathrm{TE}=306)$ seconds for the $500 \mathrm{kcal}$ TTC test. 
A summary of results from each study utilized in the current analysis, including both group responses and the incidence and proportion of non-responders, are presented in Table 1. The magnitude of individual responses for $\mathrm{VO}_{2}$ peak, relative to TE, is presented in Figure 1A. The overall rate of non-responders for $\mathrm{VO}_{2}$ peak across all participants studied was $22 \%(14 / 63)$. Interestingly, 4 adverse responders (individuals whose $\mathrm{VO}_{2}$ peak decreased by more than $2 \mathrm{x} \mathrm{TE}$ ) were observed. In the 2 studies where participants trained 4 times per week (Ma et al. 2013, Scribbans et al. 2014a) no non-responders for $\mathrm{VO}_{2}$ peak were observed. In studies where participants trained 3 times per week relatively high rates of 31\% (4/13) (Scribbans et al. 2014b), $50 \%(5 / 10 ; 30$ second SIT) and 30\% (4/12; 15 second SIT) (Zelt et al. 2014) were observed. The exception to these high rates of non-response for studies utilizing 3 training sessions per week was the study where SIT consisted of 1 minute intervals performed at $100 \%$ of maximal aerobic power (no non-responders were observed in this study) (Boyd et al. 2013). A significant correlation $(\mathrm{r}=-0.52 ; \mathrm{p}<0.001)$ was observed between individual baseline $\mathrm{VO}_{2}$ peak and the change in $\mathrm{VO}_{2}$ peak observed following training.

The incidence of non-responders for the TTC test and lactate threshold are presented in Table 1, while the relationships between the change in $\mathrm{VO}_{2}$ peak and TTC and lactate threshold are presented in Figure 1B and 1C respectively. No significant correlations were observed between the changes in $\mathrm{VO}_{2}$ peak and TTC $(\mathrm{r}=0.014 ; \mathrm{p}=0.96)$ or lactate threshold $(\mathrm{r}=0.17 ; \mathrm{p}=$ 0.44). A non-response rate of $44 \%(8 / 18)$ was observed for the TTC test while $50 \%(11 / 22)$ of participants failed to increase lactate threshold. An increase in $\mathrm{VO}_{2}$ peak was observed for all participants who failed to demonstrate an increase for TTC (Figure 2). Of the 11 individuals who demonstrated a non-response for lactate threshold, only 6 exhibited an increase in $\mathrm{VO}_{2}$ peak (Figure 3). None of the individuals demonstrating an adverse response for either $\mathrm{VO}_{2}$ peak or 
lactate threshold demonstrated an adverse response for another variable; however, 1 participant was an adverse responder for $\mathrm{VO}_{2}$ peak and a non-responder for lactate threshold (see participant 16 in Figure 3).

\section{Discussion}

The major findings from the current analysis revealed that: i) a significant proportion of individuals demonstrate a non-response following 4-6 weeks of SIT, ii) non-responders were not observed when SIT was performed 4 times per week or when intervals were performed at 100\% of maximal max aerobic power for $1 \mathrm{~min}$, and iii) individual patterns of response were observed across changes in $\mathrm{VO}_{2}$ peak and submaximal performance, however, there were 5 individuals who failed to improve either $\mathrm{VO}_{2}$ peak or lactate threshold raising the possibility that there may be global non-responders to SIT.

\section{Incidence of non-responders for $\mathrm{VO}_{2}$ peak}

The current data demonstrates a significant incidence of non-responders ( $22 \%$ overall) following 3-6 weeks of SIT. The incidence of non-response in the current study was determined using TE (Hopkins 2000), a parameter that considers both the biological variability and the technical error of measurement observed within a given laboratory and provides a robust and conservative threshold for the determination of individual response (Bouchard et al. 2012). In the present study, the overall incidence of non-response $(22 \%)$ is consistent with previous reports following both END (20-45\%) (Sisson et al. 2009, Scharhag-Rosenberger et al. 2012) and 2 weeks of SIT (35\%) (Astorino and Schubert 2014). Importantly, our results confirm a relatively high incidence of non-response following SIT when TE, rather than previously published coefficient of variations, are used to define a non-responder. While these results suggest that the 
incidence of non-response following END and SIT are likely similar, there is a need for future studies designed to specifically address this issue. Further, while our results contradict the suggestion that HIIT/SIT might induce superior individual results than END (Bacon et al. 2013), whether individuals who are insensitive to END might respond to SIT remains an important area for future study.

An important observation from the current study is the striking difference in the incidence of non-response following protocols that required participants to train three (Scribbans et al. 2014b, Zelt et al. 2014) compared to four (Ma et al. 2013, Scribbans et al. 2014a) times per week. The incidence of non-response for SIT 3 times per week was 37\%; in contrast, no nonresponders were observed when SIT was performed 4 times per week. Further, the group changes in $\mathrm{VO}_{2}$ peak when SIT was performed 4 times per week were greater than that observed following 3 times per week SIT (See Table 1), and greater than is typically observed following SIT (Gist et al. 2013, Sloth et al. 2013). These results suggest that there is a threshold of SIT training stimuli above which a greater percentage of individuals will respond. While the exact dose of SIT required to reach this threshold remains unclear, it would appear that training 4 times per week is an important consideration. Contrary to the idea that training 4 times per week is required to minimize the incidence of non-responders is the observation that participants completing a much larger dose of near maximal SIT (1 min intervals at 100\% WRpeak) 3 times per week all increased $\mathrm{VO}_{2}$ peak (Boyd et al. 2013). These observations are consistent with the findings from DREW that the incidence of non-responders decreases with increasing training dose (Sisson et al. 2009). While it must be noted that the participants completing this protocol were overweight/obese, reported being sedentary prior to starting training, and had the lowest baseline $\mathrm{VO}_{2}$ peak (which, in the current analysis was negatively associated with the change in 
$\mathrm{VO}_{2}$ peak across all participants), it is unclear if this would have impacted their response given the controversy surrounding whether baseline $\mathrm{VO}_{2}$ peak influences the magnitude of increase in $\mathrm{VO}_{2}$ peak following training (Timmons 2011).

Taken together, the finding that a negligible incidence of non-response in $\mathrm{VO}_{2}$ peak is present following SIT training four times per week, or three times per week with a relatively high SIT dose, suggests that greater exercise stimuli (i.e. increase training frequency or dose) may reduce the number of non-responders to training, perhaps by eliciting adaptation in individuals with a low sensitivity to exercise (Buford et al. 2013).

\section{Individual patterns of response following SIT}

While non-responders for $\mathrm{VO}_{2}$ peak following END have been observed, there is increasing evidence supporting individual patterns of response whereby individuals who do not demonstrate an increase in $\mathrm{VO}_{2}$ peak are likely to improve in other variables typically associated with training. For example, adverse responders for cardio-metabolic disease risk factors were found to have similar increases in $\mathrm{VO}_{2}$ peak as non-adverse responders (Bouchard et al. 2012), and few individuals demonstrate a non-response for both $\mathrm{VO}_{2}$ peak and submaximal performance (Vollaard et al. 2009, Scharhag-Rosenberger et al. 2012). The current analysis demonstrates that individual patterns of response across $\mathrm{VO}_{2}$ peak and submaximal performance are also present following SIT, a finding that is consistent with the END literature, and a previous demonstration of individual patterns of response following two weeks of SIT (Astorino and Schubert 2014). Interestingly, we have also observed a relatively high incidence $(23 \%)$ of individuals who failed to increase either $\mathrm{VO}_{2}$ peak or lactate threshold following four weeks of SIT three times per week. It is important to note, that these "global" non-responders completed SIT requiring a very low training dose (4-6, 15-30 second intervals 3 times per week), with the majority (4/5) of these 
"global non-responders" being individuals from the 15 second interval group. This provides further evidence supporting the importance of training dose for reducing the incidence of nonresponders to SIT.

An interesting consideration, which unfortunately could not be addressed in the current analysis, is the impact of individual effort on the adaptations induced by SIT. Unlike many HIIT protocols and most END protocols where a specific workload and total volume of work is prescribed in each training session, SIT allows the peak power and total work performed during each training session to vary based on individual fitness and effort. Thus, it is possible that those individuals who don't respond to SIT are simply the individuals who put in the least amount effort. While it is unlikely that this explains all individual variability following SIT, individual effort should be considered/measured in future SIT studies.

\section{Conclusion}

At present, while there is emerging evidence demonstrating the incidence of nonresponse following END, there is limited data available regarding non-response following SIT. The current analysis demonstrates significant incidence of non-responders for $\mathrm{VO}_{2}$ peak following SIT. Further, we confirmed a similar heterogeneity in the individual patterns of response following SIT and following END. Importantly, the incidence of non-response was highest following SIT protocols that required the lowest training volume, highlighting the importance of training dose (i.e. training session energy expenditure and/or training frequency) and supporting the potential that incidence of non-response may be reduced once the trainingdose threshold of SIT is reached. Future work is needed to both compare incidences of nonresponse following END and SIT, to compare the incidence of non-response to SIT in men and women, and to determine the optimal dose of SIT for minimizing individual non-response. 
These results contribute to a growing awareness of individual responsiveness to exercise training by demonstrating a significant rate of non-response following SIT and by highlighting the potential impact optimization of SIT (i.e. training frequency/duration) might have on minimizing the risk of non-response following exercise prescription.

\section{Conflict of Interest}

The authors declare that there are no conflicts of interests associated with this manuscript. 


\section{Reference List}

Astorino, T. A., and Schubert, M.M. 2014. Individual responses to completion of short-term and chronic interval training: A retrospective study. PLoS One 9(5). doi: 10.1371/journal.pone.0097638.

Bacon, A.P., Carter, R.E., Ogle, E. A., and Joyner, M.J. 2013. VO2max Trainability and High Intensity Interval Training in Humans: A Meta-Analysis. PLoS One 8(9). doi: 10.1371/journal.pone.0073182.

Bishop, D., Jenkins, D.G., and Mickinnon, L.T. 1998. The relationship between plasma lactate parameters, Wpeak and 1-h cycling performance in women. Med. Sci. Sports Exerc. 30(8): $1270-1275$.

Bouchard, C., Blair, S.N., Church, T.S., Earnest, C.P., Hagberg, J.M., Häkkinen, K., Jenkins, N.T., Karavirta, L., Kraus, W.E., Leon, A.S., Rao, D.C., Sarzynski, M. A., Skinner, J.S., Slentz, C. A., and Rankinen, T. 2012. Adverse metabolic response to regular exercise: Is it a rare or common occurrence? PLoS One 7(5). doi: 10.1371/journal.pone.0037887.

Boyd, J.C., Simpson, C. A., Jung, M.E., and Gurd, B.J. 2013. Reducing the Intensity and Volume of Interval Training Diminishes Cardiovascular Adaptation but Not Mitochondrial Biogenesis in Overweight/Obese Men. PLoS One 8(7): 1-8. doi:

10.1371/journal.pone.0068091.

Buford, T.W., Roberts, M.D., and Church, T.S. 2013. Toward exercise as personalized medicine. Sport. Med. 43(3): 157-165. doi: 10.1007/s40279-013-0018-0.

Edgett, B. A., Foster, W.S., Hankinson, P.B., Simpson, C. A., Little, J.P., Graham, R.B., and Gurd, B.J. 2013a. Dissociation of Increases in PGC-1 $\alpha$ and Its Regulators from Exercise Intensity and Muscle Activation Following Acute Exercise. PLoS One 8(8). doi: 10.1371/journal.pone.0071623.

Edgett, B. A., Ross, J.E.D., Green, A.E., MacMillan, N.J., Milne, K.J., and Gurd, B.J. 2013 b. The effects of recreational sport on VO2peak, VO2 kinetics and submaximal exercise performance in males and females. Eur. J. Appl. Physiol. 113: 259-266. doi: $10.1007 / \mathrm{s} 00421-012-2435-4$.

Esfarjani, F., and Laursen, P.B. 2007. Manipulating high-intensity interval training: Effects on VO2 max, the lactate threshold and $3000 \mathrm{~m}$ running performance in moderately trained males. J. Sci. Med. Sport 10: 27-35. doi: 10.1016/j.jsams.2006.05.014.

Gibala, M.J., Little, J.P., van Essen, M., Wilkin, G.P., Burgomaster, K. A, Safdar, A., Raha, S., and Tarnopolsky, M. A. 2006. Short-term sprint interval versus traditional endurance 
training: similar initial adaptations in human skeletal muscle and exercise performance. J. Physiol. 575(2006): 901-911. doi: 10.1113/jphysiol.2006.112094.

Gillen, J.B., and Gibala, M.J. 2014. Is high-intensity interval training a time-efficient exercise strategy to improve health and fitness? Appl. Physiol. Nutr. Metab. 39(3): 409-12. doi: 10.1139/apnm-2013-0187.

Gist, N.H., Fedewa, M. V, Dishman, R.K., and Cureton, K.J. 2013. Sprint Interval Training Effects on Aerobic Capacity: A Systematic Review and Meta-Analysis. Sports Med. (44): 269-279. doi: 10.1007/s40279-013-0115-0.

Hopkins, W.G. 2000. Measures of reliability in sports medicine and science. Sports Med. 30(1): 1-15. doi: 10.2165/00007256-200030050-00006.

Jeukendrup, A, Saris, W.H., Brouns, F., and Kester, A.D. 1996. A new validated endurance performance test. doi: 10.1097/00005768-199602000-00017.

Ma, J.K., Scribbans, T.D., Edgett, B.A., Boyd, J.C., Simpson, C.A., Little, J.P., and Gurd, B.J. 2013. Extremely low-volume, high-intensity interval training improves exercise capacity and increases mitochondrial protein content in human skeletal muscle. Open J. Mol. Integr. Physiol. 03(November): 202-210. doi: 10.4236/ojmip.2013.34027.

MacPherson, R.E.K., Hazell, T.J., Olver, T.D., Paterson, D.H., and Lemon, P.W.R. 2011. Run sprint interval training improves aerobic performance but not maximal cardiac output. Med. Sci. Sports Exerc. 43: 115-122. doi: 10.1249/MSS.0b013e3181e5eacd.

Scharhag-Rosenberger, F., Walitzek, S., Kindermann, W., and Meyer, T. 2012. Differences in adaptations to 1 year of aerobic endurance training: Individual patterns of nonresponse. Scand. J. Med. Sci. Sport. 22(1): 113-118. doi: 10.1111/j.1600-0838.2010.01139.x.

Scribbans, T.D., Edgett, B.A., Vorobej, K., Mitchell, A.S., Joanisse, S.D., Matusiak, J.B.L., Parise, G., Quadrilatero, J., and Gurd, B.J. 2014a. Fibre-specific responses to endurance and low volume high intensity interval training: Striking similarities in acute and chronic adaptation. PLoS One 9(6). doi: 10.1371/journal.pone.0098119.

Scribbans, T.D., Ma, J.K., Edgett, B.A., Vorobej, K.A., Mitchell, A.S., Zelt, J.G.E., Simpson, C.A., Quadrilatero, J., and Gurd, B.J. 2014b. Resveratrol supplementation does not augment performance adaptations or fibre-type specific responses to high-intensity interval training in humans. Appl. Physiol. Nutr. Metab. 1313(July): 1305-1313.

Sisson, S.B., Katzmarzyk, P.T., Earnest, C.P., Bouchard, C., Blair, S.N., and Church, T.S. 2009. Volume of exercise and fitness nonresponse in sedentary, postmenopausal women. Med. Sci. Sports Exerc. 41(3): 539-545. doi: 10.1249/MSS.0b013e3181896c4e. 
Sloth, M., Sloth, D., Overgaard, K., and Dalgas, U. 2013. Effects of sprint interval training on VO2max and aerobic exercise performance: A systematic review and meta-analysis. Scand. J. Med. Sci. Sport. 23(6): 341-352. doi: 10.1111/sms.12092.

Timmons, J.A. 2011. Variability in training-induced skeletal muscle adaptation. J. Appl. Physiol. 110(3): 846-853. doi: 10.1152/japplphysiol.00934.2010.

Vollaard, N.B.J., Constantin-Teodosiu, D., Fredriksson, K., Rooyackers, O., Jansson, E., Greenhaff, P.L., Timmons, J.A, and Sundberg, C.J. 2009. Systematic analysis of adaptations in aerobic capacity and submaximal energy metabolism provides a unique insight into determinants of human aerobic performance. J. Appl. Physiol. 106(5): 1479-1486. doi: 10.1152/japplphysiol.91453.2008.

Zelt, J.G.E., Hankinson, P.B., Foster, W.S., Williams, C.B., Reynolds, J., Garneys, E., Tschakovsky, M.E., and Gurd, B.J. 2014. Reducing the volume of sprint interval training does not diminish maximal and submaximal performance gains in healthy men. Eur. J. Appl. Physiol. doi: 10.1007/s00421-014-2960-4. 
Table 1. Baseline and training induced changes in VO2peak, lactate threshold and time to completion (TTC) for the 5 studies included in the current analysis.

\begin{tabular}{|c|c|c|c|c|}
\hline \multirow[b]{2}{*}{ Pre } & \multirow[b]{2}{*}{ Post } & \multirow[b]{2}{*}{ Delta } & \multicolumn{2}{|c|}{$\begin{array}{c}\text { Non/Adverse- } \\
\text { Responders }\end{array}$} \\
\hline & & & $\mathbf{N}$ & $\%$ \\
\hline
\end{tabular}

\section{Boyd (2013)}

1 min intervals $100 \%$ of WRpeak $3 \mathrm{x} /$ week for 3 weeks

$\begin{array}{rccccc}\mathrm{VO}_{2} \text { peak } & 35.4(5.4) & 44.7(5.0)^{*} & 9.4(3.1) & 0 & 0 \\ \mathrm{TTC} & 2365(598) & 2034(532)^{*} & -331(123) & 4 & 44\end{array}$

\section{Ma (2013)}

$20 \mathrm{sec}$ intervals

$\mathrm{VO}_{2}$ peak $39.7(3.0)$

$47.2(2.9)^{*} \quad 7.5(3.2)$

0

0

$4 \mathrm{x} /$ week for 4 weeks

\section{Scribbans (2014a)}

20 sec intervals $170 \%$ WRpeak $4 \mathrm{x} /$ week for 6 weeks

$$
\begin{array}{rc}
\mathrm{VO}_{2} \text { peak } & 48.3(6.1) \\
\mathrm{TTC} & 2215(210)
\end{array}
$$

$1881(173)^{*} \quad-334(82)$

\section{0}

4

0

\section{Scribbans (2014b)}

$20 \mathrm{sec}$ intervals $170 \%$ WRpeak

$$
\mathrm{VO}_{2} \text { pea }
$$

$50.4(5.0)$

$53.3(5.4)^{*} \quad 3.0(3.3)$

4

31

$3 \mathrm{x} /$ week for 3 weeks

\section{Zelt (2014)}

$30 \mathrm{sec}$ intervals All-out intensity $3 \mathrm{x} /$ week for 4 weeks

$$
\begin{array}{rc}
\mathrm{VO}_{2} \text { peak } & 49.8(7.8) \\
\mathrm{LT} & 198(30)
\end{array}
$$

$$
51.5(8.1)
$$

$1.71(3.9)$

5
3

50

$221(32)$

$23(26)$

$30 \mathrm{sec}$ intervals All-out intensity $3 \mathrm{x} /$ week for 4 weeks

$\begin{array}{rccccc}\mathrm{VO}_{2} \text { peak } & 44.2(8.7) & 47.1(7.6)^{*} & 3.0(4.4) & 4 & 30 \\ \mathrm{LT} & 200(35) & 215(32) & 15(29) & 8 & 67\end{array}$

\section{TOTAL}

$\begin{array}{rr}\mathrm{VO}_{2} \text { peak } & 46.1(8.4) \\ \text { TTC } & 2280(443) \\ \text { LT } & 199(32)\end{array}$

$\begin{array}{cc}50.5(7.4) & 4.4(4.4) \\ 1945(396) & -334(100) \\ 217(31) & 19(27)\end{array}$

14
8
11

Values are presented as Mean ( $\pm \mathrm{SD}) . \mathrm{VO}_{2}$ peak $(\mathrm{mL} / \mathrm{kg} / \mathrm{min}) ; \mathrm{TTC}$, time to completion (seconds); LT, lactate threshold (watts); N, number of non/adverse responders; \%, the proportion of non/adverse responders from each participant pool.

* Significant $(p>0.05)$ effect of training within group. 


\section{Figure Captions}

Figure 1. Individual participant responses to SIT from all studies are shown (A) with $2 \mathrm{x}$ the TE illustrated using dashed lines. Non-responders have a $\mathrm{VO}_{2}$ peak response that falls within $2 \mathrm{x}$ the TE (shaded region), while adverse responders (checkered bars) have a decrease in $\mathrm{VO}_{2}$ peak greater than $2 \mathrm{x}$ the TE. The relationships between individual changes in $\mathrm{VO}_{2}$ peak and $500 \mathrm{kcal}$ TTC (B; Boyd [2013] open circles; Scribbans [2014a] closed circles) or lactate threshold (C; 30 second intervals open circles; 15 second intervals closed circles) are also shown. Dashed lines on panel $\mathrm{B}$ and $\mathrm{C}$ represent the typical error, while an individual falling within the shaded area would have demonstrated a non-response for both variables.

Figure 2. Individual incidence of response for $\mathrm{VO}_{2}$ peak and TTC are also shown with nonresponders (grey).

Figure 3. Individual incidence of response for $\mathrm{VO}_{2}$ peak and lactate threshold are also shown with non-responders (grey), adverse responders (checkered) and overall non-responders (black). 
Figure 1

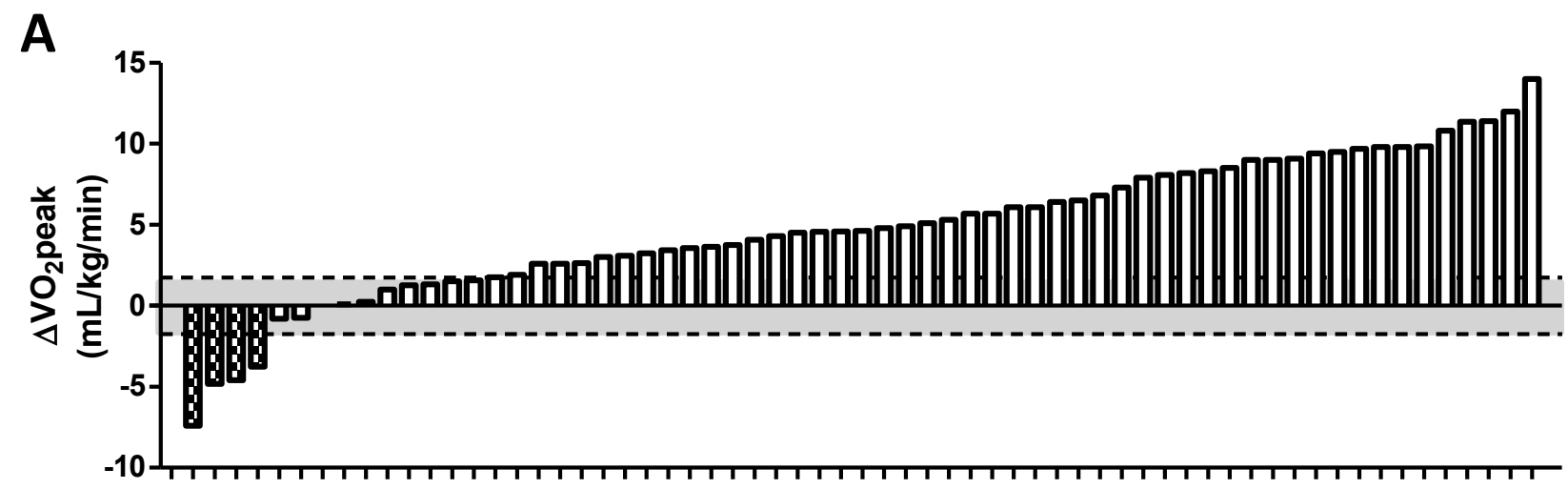

Individual Participant Responses

B

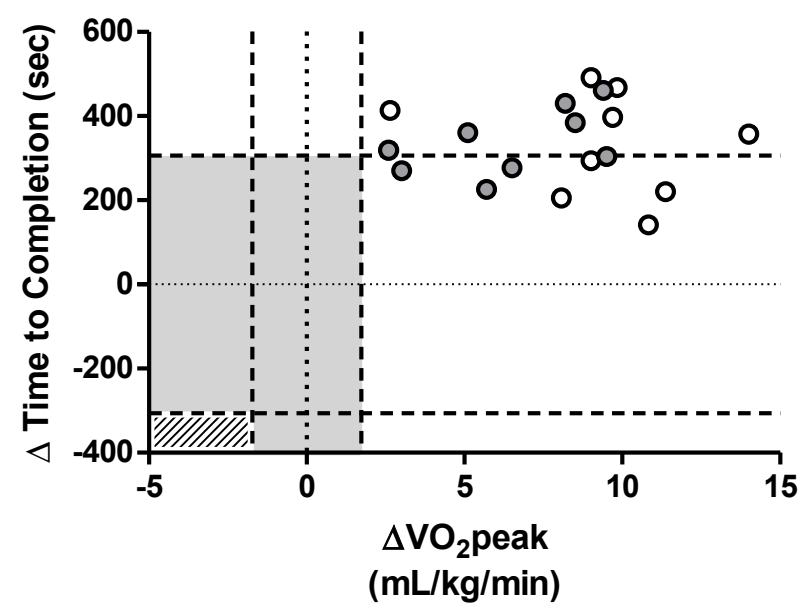

C

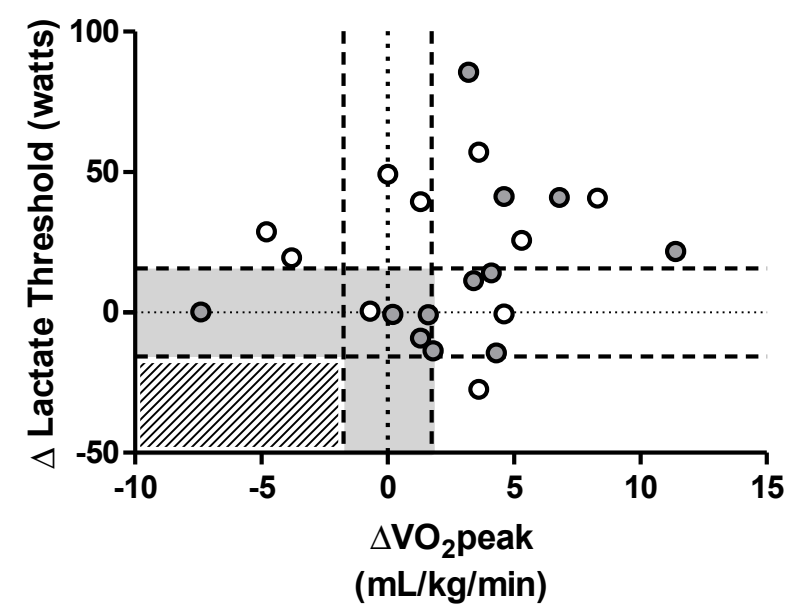


Figure 2

\begin{tabular}{|l|c|c|c|c|c|c|c|c|c|c|c|c|c|c|c|c|c|c|c|}
\hline & \multicolumn{10}{|c|}{ Boyd (2013) } & \multicolumn{10}{|c|}{ Scribbans (2014a) } & \\
\hline & 1 & 2 & 3 & 4 & 5 & 6 & 7 & 8 & 9 & 10 & 11 & 12 & 13 & 14 & 15 & 16 & 17 & 18 & Total \\
\hline VO $_{2}$ peak & & & & & & & & & & & & & & & & & & & $0(0 \%)$ \\
\hline TTC & & & & & & & & & & & & & & & & & & & $8(44 \%)$ \\
\hline \multicolumn{1}{|c|}{ OVERALL } & & & & & & & & & & & & & & & & & & & $0(0 \%)$ \\
\hline
\end{tabular}


Figure 3

\begin{tabular}{|c|c|c|c|c|c|c|c|c|c|c|c|c|c|c|c|c|c|c|c|c|c|c|c|}
\hline & \multicolumn{10}{|c|}{30 second intervals } & \multicolumn{12}{|c|}{15 second intervals } & \multirow[b]{2}{*}{ Total } \\
\hline & 1 & 2 & 3 & 4 & 5 & 6 & 7 & 8 & 9 & 10 & 11 & 12 & 13 & 14 & 15 & 16 & 17 & 18 & 19 & 20 & 21 & 22 & \\
\hline $\mathrm{VO}_{2}$ peak & & & & & & & & & & & & & & & & & & & & & & & 9 (41\%) \\
\hline Lactate Threshold & & & & & & & & & & & & & & & & & & & & & & & $11(50 \%)$ \\
\hline OVERALL & & & & & & & & & & & & & & & & & & & & & & & $5(23 \%)$ \\
\hline
\end{tabular}

